\title{
Kommission für Erwerbung und Bestandsentwicklung des EDBI Die Versorgung der Hochschulen mit wissenschaftlicher Literatur Adalbert Kirchgäßner
}

Die wissenschaftlichen Bibliotheken befinden sich derzeit in einer Krise, die durch den steigenden Bedarf an wissenschaftlichen Informationen einerseits und durch kaum wachsende, teilweise stagnierende und reduzierte Etats offenkundig wurde.

\section{Probleme der Literaturversorgung}

- Wissenschaftliche Zeitschriften sind nicht nur Informationsträger, die ihren bestimmten Preis haben. Sie haben innerhalb der scientific community als spezifische Publikationsform einen von Titel, Verlag, und Editor abhängigen sozialen Mehrwert, der ganz maßgeblich auch über die wissenschaftliche Reputation, die Karriere, die Höhe von Forschungsgeldern und den akademischen Status ihrer Autoren entscheidet. Das Publikationsverhalten der wissenschaftlichen Nutzer einer Bibliothek wird u.a. durch nur vordergründig immaterielle (soziale und berufs- und individualpsychologische) Überlegungen beeinflusst, die sich gleichwohl zu Lasten der Bibliothek auch in der Höhe der vom Prestigewert der Publikationsform 'Zeitschrift' bestimmten Abonnementskosten niederschlagen. Dieser soziale Mehrwert wird derzeit durch einen kostengünstig auf einem Dokumentenserver abgelegten und nicht in eine Publikationsform eingebetteten Artikel nicht erzielt.

- Der (zum Teil überteuerte) Rückkauf der bereits intensiv mit öffentlichen Geldern geförderten wissenschaftlichen Informationen durch die öffentliche Hand ist Indikator dafür, dass die Finanzkrise der Bibliotheken auch durch strukturelle hochschulrechtliche und förderungspolitische Vorgaben auf dem Nachfragemarkt bedingt ist.

- Die Digitalisierung der Informationsträger verringert offenbar nicht, was anzunehmen gewesen wäre, die Nachfrage nach gedruckten Zeitschriften. Sie verringert offenbar auch nicht das Fernleihaufkommen. Sie erhöht vielmehr generell die Nachfrage der Nutzer, den artikulierten Bedarf des Nachfragemarktes und damit die Anforderungen und den finanziellen Aufwand der Bibliotheken zu dessen Befriedigung.

- Die Bibliotheken befinden sich in einer Finanzkrise. Über die Ursachen der Finanzkrise besteht weitgehend Konsens: mit kaum wachsenden, stagnie- 
renden oder reduzierten Etats können Preissteigerungen vor allem der Zeitschriftenverlage und dort vor allem im STM-Bereich, ungünstige Wechselkursentwicklungen, eine wachsende Wissenschaftsproduktion und der Einkauf neuer Produktgruppen im Bereich der digitalen Medien nicht aufgefangen werden. Um finanziell handlungsfähig zu bleiben, wird versucht, die Fixkosten durch Zeitschriftenabbestellungen zu reduzieren.

- Die durch diese Maßnahmen zu erzielenden Spareffekte greifen nur kurzfristig und sind derzeit in einem, spätestens in zwei Jahren durch die proportional zur Abbestellquote steigenden Preise für die Abonnements der verbliebenen Zeitschriften aufgefressen.

- Der Wissenschaftsstandort Deutschland ist gefährdet. Anzunehmen ist, dass zwischen den beklagten zu langen Studienzeiten und der Abwanderung von Wissenschaftlern ins Ausland und der unzureichenden Literaturversorgung ein Zusammenhang besteht.

- Die Deutsche Bibliotheksstatistik zeigt den Rückgang der Zugangsmengen an Monographien und Zeitschriftenbeständen auf, auch wenn sie Zahlen über fehlende Abonnements, abbestellte Titel und nicht beschaffte Monographien nicht nachweisen kann. Die in der Vergangenheit im Auftrag der DFG von Rolf Griebel erstellten Analysen zur Etatsituation der wissenschaftlichen Bibliotheken fortzuführen, ist ein dringendes Desiderat. Die Behinderung der Wissenschaft durch die mangelnde Informationsversorgung ist im täglichen Betrieb der Bibliotheken offenkundig.

- Die durch Abkehr vom Print-Medium und der Bereitstellung digitalisierter Informationen erwarteten finanziellen Entlastungseffekte treten nicht ein, weder bei den Personalkosten noch im Erwerbungsetat. Laufzeitklauseln, Abbestellklauseln, garantierte Preissteigerungsklauseln, erhöhte Mehrwertsteuersätze für Electronic-only, die Bindung der Preisgestaltung von elektronischen Informationsträgern an Print-Holdings, fehlende Preismodelle für Pay-per-View und die Präferenz der Verlage, bei Konsortiallösungen möglichst komplette Verlagspakete anzubieten, sind Indikatoren dafür, dass die Finanzkrise der Bibliotheken nicht nur durch pekuniär-finanzielle, sondern auch durch strukturelle, steuer-, rechts- und vertriebspolitische Vorgaben auf dem Beschaffungsmarkt bedingt ist. Die technische Revolution der prinzipiellen globalen zeitlichen und räumlichen Verfügbarkeit aller Informationen für jedermann per Internet ist Theorie und wird in der Praxis durch ausgeklügelte Zugriffsbeschränkungen konterkariert.

- Der Nachfragemarkt der wissenschaftlichen Bibliotheken besteht aus den Produzenten von Wissenschaft. Der Etat der Bibliotheken hält mit den bei diesen Produzenten zu konstatierenden Steigerungsraten in puncto Forschungsförderung und Forschungsintensität nicht mit. Der Seitenumfang 
der Zeitschriften wächst, desgleichen die Zahl und der Spezialisierungsgrad der Zeitschriftentitel und damit deren Preis.

\section{Stand der Diskussion}

Die seit einigen Jahren wachsenden Schwierigkeiten der wissenschaftlichen Bibliotheken, mit den vorhandenen Mitteln ihrem Literaturversorgungsauftrag nachzukommen, waren auch auf dem 91. Deutschen Bibliothekartag in Bielefeld im April 2001 Thema auf einer von den Veranstaltern abgehaltenen Pressekonferenz. Eine dort vor der Presseöffentlichkeit vorgetragene pointierte Stellungnahme zur Ursache und den Hintergründen der sich zu einer Bibliothekskrise ausweitenden Etatengpässe durch den Leitenden Bibliotheksdirektor der UB Bielefeld, Karl Wilhelm Neubauer, führte zu einem Austausch Offener Briefe zwischen K.W. Neubauer und dem VDB ${ }^{1}$ und zu einer Gegenerklärung der $\mathrm{BDB}^{2}$. Der Berufsverband Information Bibliothek (BIB) äußerte sich gesondert ${ }^{3}$. Bereits 1999 hatte die Erwerbungskommission in einem Offenen Brief an führende Zeitschriftenverlage auf die Preisspiralenproblematik im Zeitschriftenbereich aufmerksam gemacht ${ }^{4}$. Parallel zu diesem Diskussionsstrang hat der Verein Forum Zeitschriften / GeSIG in einem an die Bundestagsfraktionen gerichteten Brief an deren politische Verantwortung und an seine im Vorjahr der KMK überreichte Unterschriftenaktion erinnert und angesichts der Gefährdung des Wissenschaftsstandorts Deutschland Unterstützung für die Bibliotheken angemahnt ${ }^{5}$. Der Deutsche Kulturrat hat in einer Resolution an Bundes- und Landesregierungen appelliert, ausreichende Gelder zur Verfügung zu stellen, um das für Forschung, Lehre und Studium unabdingbare Titelvolumen an wissenschaftlichen Zeitschriften halten zu können $^{6}$. Die Initiative des Kulturrats ist von der FDP-Fraktion aufgegriffen worden und hat zu einem in den Deutschen Bundestag eingebrachten Antrag auf Verabschiedung eines Sonder- und Soforthilfeprogramms mit Bereitstellung zusätzlicher Mittel geführt. Aus Sicht der BDB ist zum ersten Mal im Bundestag über das Bibliothekswesen in dieser Ausführlichkeit debattiert worden. Der An-

1 Vgl. vdb-Mitteilungen 2001, H. 2, S. 4-7 und BiBLIOTHEKSDIENST 35(2001),H. 9, S. $991-993$

2 Vgl. BiBliOtheKSDIENSt 35 (2001), S. 679-683.

3 Vgl. Böttger, Klaus-Peter: Betr.: Zukunft der Bibliotheken. In: Buch und Bibliothek. 53 (2001), S. 436

4 Zeitschriftenpreise 1999 - Offener Brief. In: BIBLIOTHEKSDIENST 33 (1999),S. 311313.

5 Vgl. BiBliOtheKSDIENSt 35 (2001), S. 1351-1353.

6 Vgl. Hochschulbibliotheken bluten aus - Deutschland verliert international den Anschluss. http://www.kulturrat.de 
trag der FDP-Fraktion wurde an die zuständigen Ausschüsse verwiesen ${ }^{7}$. Die Arbeitsgruppe 'Bibliotheken' der KMK hat eine ad-hoc-AG Bibliothekskrise eingesetzt und sich in ihrer Novembersitzung mit dem Thema befasst. Die Hochschulrektorenkonferenz beabsichtigt ebenfalls, eine Arbeitsgruppe einzurichten, um Strategien zur Überwindung der Etatkrise der wissenschaftlichen Bibliotheken zu entwickeln. Und schließlich hat der Wissenschaftsrat in seinen jüngsten Empfehlungen grundsätzliche Aussagen zur Rolle der Bibliotheken und der dafür erforderlichen Etatausstattung in der Informationsgesellschaft gemacht ${ }^{8}$.

Der DBV beabsichtigt, in den laufenden Diskussionsprozess einzugreifen und seinerseits ebenfalls zur Etatkrise der wissenschaftlichen Bibliotheken Stellung zu nehmen. Er ist an die Kommission für Erwerbung und Bestandsentwicklung des EDBI mit der Bitte herangetreten, aus ihrer Sicht und des in der Kommission vorhandenen Erkenntnisstandes einen Beitrag zu leisten. Mit den bereits vorliegenden, oben zitierten Resolutionen, Diskussionsbeiträgen und Stellungnahmen liegt bereits ein ganzes Bündel von Analysen, Argumenten, Forderungen und strategischen Überlegungen vor, die in ihren einzelnen Aspekten von der Erwerbungskommission zum Teil unterschiedlich bewertet und gewichtet, aber deren Kernaussagen grundsätzlich richtig sind. Der vorliegende Beitrag der Erwerbungskommission will deshalb nicht bisher bereits mehrfach Gesagtes noch einmal wiederholend bestätigen und in pauschalisierende Forderungen nach mehr Unterstützung ummünzen. Die vorangehenden Thesen und die folgenden Ausführungen dazu stellen die Finanzkrise der Bibliotheken in den Zusammenhang mit der Systemkrise im Informationsprozess, nämlich dem Prozess der Produktion und Reproduktion von Wissenschaft, an dem es mehrere verschiedene Systembeteiligte gibt. Mehr Beteiligte jedenfalls als nur jene drei, die pauschal abgestempelt derzeit im Blickfeld der Diskussion stehen: die Unterhaltsträger (Stempel: „mehr Geld“), die Bibliotheken (Stempel: „Strukturelle Defizite“) und die Verlage (Stempel: „Preistreiber“). Wesentliche Teilnehmer in diesem Prozess sind die Wissenschaftler als

- Produzenten wissenschaftlicher Erkenntnisse,

- Herausgeber und Gutachter zur Sicherung Qualität wissenschaftlicher Publikationen,

- Autoren wissenschaftlicher Publikationen und

7 Deutscher Bundestag. 14. Wahlperiode. 162. Sitzung. Stenographischer Bericht. In: Plenarprotokoll 14/162 vom 30. März 2001 sowie Drucksache 14/5105 vom 17.01.2001.

8 Wissenschaftsrat: Empfehlungen zur digitalen Informationsversorgung durch Hochschulbibliotheken. Drs. 4935/01 vom 13.7.2001, insbesondere Abschnitte B III bis V. 
- Nutzer eben dieser wissenschaftlichen Publikationen.

\section{Handlungsbedarf}

- Die durch fehlende Erwerbungsmittel charakterisierte „Bibliothekskrise“ beruht nur vordergründig auf einer Finanzkrise. Vielmehr sind die finanziellen Probleme Ausdruck einer grundlegenden Strukturkrise ${ }^{9}$, aber nicht oder doch nicht allein der Bibliotheken, wie K.W. Neubauer behauptet, sondern der derzeit etablierten und beherrschenden Strukturen bei der Produktion, Distribution und Rezeption von Wissenschaft und zwar unabhängig vom gewählten (digitalisierten oder print)Medium.

- Die alleinige Forderung nach und die Bereitstellung von „Mehr Geld“ löst die Bibliothekskrise allenfalls kurzfristig. Sie ändert nicht das Publikationsverhalten der Autoren, die Vertriebsstrategien der Verlage, das Nachfrageverhalten der Nutzer, oder die Informationsvermittlungsusancen der Bibliotheken.

- Die Bibliothekskrise ist nur durch Einbindung aller Systembeteiligten und nur in einem mittelfristig angelegten Prozess zu überwinden, der auf Verhaltensänderung zielt. Diese Verhaltensänderungen sind vermutlich nur über den Hebel „Geld“ zu erreichen (auf eine einfache Formel gebracht: Geldmangel = Informationsmangel = Zwang zur Änderung der Informationsbeschaffung).

- Auf der Ebene der Produzenten von Wissenschaft, der Autoren also, ließen sich über vertrags- und hochschulrechtliche Regelungen Verfahren zu entwickeln, die es verbieten, die Nutzungsrechte wissenschaftlicher Arbeiten von vornherein zu kommerzialisieren und dauerhaft allein auf Gewinnmaximierung zielenden Verlagen zu übereignen. Die Verfahren zur Bewertung der Qualität von Wissenschaft über den impact factor von Zeitschriften wäre zu hinterfragen und ggf. zu korrigieren. Der Status- und Prestigewert der Publikationsform 'Zeitschrift' wäre von neuen, anderen und gleichwertigen, aber in geringere Finanzzwänge führenden Statussymbolen abzulösen.

- Auf der Ebene der Nutzer von wissenschaftlicher Information wäre als Steuerungselement über eine Kostenbeteiligung von Endnutzern bei Zugriff auf gebührenpflichtige Datenbanken ${ }^{10}$ nachzudenken, ohne damit das Prinzip der Chancengleichheit und den Verfassungsauftrag der Meinungs- und Informationsfreiheit zu verletzen.

9 Neubauer, Karl Wilhelm: Offener Brief an die Vorstände von VDB, BIB DBV. In: vdbMitteilungen 2001, H. 2, S. 4f.S. 5

10 Wissenschaftsrat: Empfehlungen zur digitalen Informationsversorgung. S. 44. 
- Auf der Ebene der Unterhaltsträger wäre bei all ihren Maßnahmen zur Entwicklung neuer Studiengänge und Forschungsfelder, zur Förderung von elektronischer Information, von Wissenschaft und von Kommunikationstechnologie (Stichwort: Global Info) sicherzustellen, dass ein Bruchteil der Fördermittel für den Erwerb und die dauerhafte Bereitstellung der aus den Förderprogrammen erwachsenen Inhalte garantiert bleibt. Forschungsrelevante Fortschritte dank Informationstechnologie und dank Multimedia sind nur bei gleichzeitiger Verfügbarkeit von mehr Information, Wissen und Inhalten (von mehr „content") zu erzielen.

- Auf der Ebene der Verlage, die naturgemäß rückläufige Umsätze und Gewinnmargen nicht werden anstreben können, ist ein Umdenken in der Verlags- und Vertriebspolitik nur durch den Aufbau von Konkurrenzszenarien zu erzielen: deren derzeit noch vorhandene Monopolstellung (keine Zeitschrift ist aufgrund ihrer singulären Inhalte durch eine andere substituierbar) wäre durch die verstärkte Akzeptanz alternativer nichtkommerzieller Publikationsmodelle (Stichwort: SPARC) zu korrigieren.

- Auf der Ebene der Bibliotheken wäre den veränderten Marktmechanismen (z.B. Ausschalten des Zwischenhandels durch die Verlage und Direktvertrieb zumindest der elektronischen Publikationen) durch länderübergreifenden koordinierten Einkauf und Konsortiallösungen mit professioneller Verhandlungsführerschaft Rechnung zu tragen. Derzeit führt der konsortiale Einkauf von E-Medien jedoch nur bei Datenbanken, nicht aber bei EZeitschriften zu den Bibliotheksetat entlastenden Preisnachlässen. Bei EZeitschriften verfügt die Bibliothek bei unverändert hohem nicht rabattiertem Preis statt dessen im Regelfall „nur“ über mehr Inhalt (= bislang nicht abonnierte Titel). 